\title{
Fragmented QRS in inferior leads is associated with non-alcholic fatty liver disease, body-mass index, and interventricular septum thickness in young men
}

ORIGINAL INVESTIGATION

\begin{abstract}
Objective: Fragmented QRS (fQRS) has been shown to be related to coronary heart disease, heart failure, hypertension, cardiac arrhythmia, and metabolic syndrome. Although fQRS in lateral leads is shown to be associated with a poor outcome in patients with a known cardiac disease, the knowledge about the significance and prevalence of $f Q R S$ in inferior leads is scarce. This study aimed to investigate the prevalence and predictors of fQRS in inferior leads in healthy young men.
\end{abstract}

Methods: A total of 1,155 men underwent electrocardiography (ECG), hepatic ultrasonography, and routine biochemical tests. A total of 210 eligible men with $\mathrm{fQRS}$ in inferior leads (group 1) and 770 eligible men without fQRS in inferior leads (group 2) were compared with each other in terms of clinical, demographic, and laboratory parameters.

Results: The prevalence of fQRS in inferior leads was found to $21.4 \%$. Body mass in$\operatorname{dex}(B M I)$, systolic blood pressure (BP), creatinine, and alanine aminotransferase levels; non-alcoholic fatty liver disease (NAFLD) percentage; and interventricular septum thickness (IVST) were significantly greater in group 1 than those in group 2. BMI, IVST, NAFLD, creatinine, ALT, and systolic BP were entered in a model of multiple regression analyses to predict $f Q R S$, a dependent variable. NAFLD was the best independent predictor of $f Q R S$ $(\beta=6.115, p=0.001)$. BMI $(\beta=1.448, p=0.014)$ and IVST $(\beta=1.058, p=0.029)$ were the other independent predictors of $\mathrm{fQRS}$ in inferior leads.

Conclusion: This study demonstrated the association of fQRS in inferior leads with NAFLD, BMI, and IVST in young men.

Keywords: body mass index, electrocardiography, non-alcoholic fatty liver disease, echocardiograghy

\section{INTRODUCTION}

Fragmented QRS (fQRS) is defined as the presence of $R^{\prime}$ wave or notching of $R$ or $S$ patterns with or without $Q$ waves on a 12-lead resting electrocardiography (ECG) (1). It indicates heterogeneous depolarization of the ventricular myocardium that can occur owing to ischemia, fibrosis, or scar. Recent studies have shown the relationship of $f Q R S$ with various cardiovascular diseases such as coronary heart disease (CHD), dilated cardiomyopathy, heart failure, hypertension, cardiac arrhythmias, and metabolic syndrome (2-9).

Although fQRS in lateral leads is shown to be associated with a poor outcome in patients with a known cardiac disease, the knowledge about the significance and prevalence of $f Q R S$ especially in inferior leads is scarce (3). A limited number of studies have reported that the prevalence of $f Q R S$ in healthy individuals ranges from $7.2 \%$ to $19.7 \%$, with the conclusion that fQRS in inferior leads is not associated with poor clinical outcomes, especially in healthy individuals $(10,11)$.

This study aimed to investigate the prevalence and predictors of fQRS in inferior leads in healthy young men.

\section{Remzi Sarıkaya (D) \\ Cihan Șengül (D) \\ Ömer Kümet (D) \\ Gürkan Imre (D) \\ Tayyar Akbulut (D) \\ Mustafa Oğuz (D)}

Department of Cardiology, University of Health Sciences, Van Training and

Research Hospital; Van-Turkey

Corresponding Author:

Remzi Sarıkaya

$\bowtie$ drremzisarikaya@gmail.com

Accepted: July 16, 2021 Available Online Date: February 2, 2022

Cite this article as: Sarıkaya $R$, Șengül C, Kümet Ö, İmre G, Akbulut T, Oğuz M. Fragmented QRS in inferior leads is associated with non-alcholic fatty liver disease, body-mass index, and interventricular septum thickness in young men. Anatol J Cardiol 2022; 26: 100-4.

DOI: 10.5152/AnatolJCardiol.2021.433 


\section{METHODS}

\section{Study population}

Recruitment to the study was conducted according to the principles outlined in the Helsinki Declaration for investigations in human subjects. The Ethics Committee approved the protocol. The participants gave written informed consent. The study population consisted of outpatient men who were referred to our army check-up center for general young health screening from January 2020 to December 2020. A total of 1,155 men underwent ECG, transthoracic echocardiography (TTE), blood pressure (BP) measurement, hepatic ultrasonography (USG), and routine biochemical tests during the specified period. Participants with a history of CHD or valvular heart disease, hypertension, systolic heart failure, diabetes mellitus, chronic kidney disease, chronic liver disease other than steatosis, excessive alcohol drinking, and hypothyroidism or hyperthyroidism were excluded from the study population. The age, height, and weight of the participants were recorded. Body mass index (BMI) was calculated by dividing body weight in kilograms by the square of the height in meters $\left(\mathrm{kg} / \mathrm{m}^{2}\right)$. The systolic BP and diastolic BP of all the participants were measured with a sphygmomanometer after at least 15 minutes of resting. Participants' cigarette exposure was questioned and cigarette exposure was defined as active smokers and former smokers who had quit smoking for less than 2 years. Current or past excessive alcohol drinking was defined by an average daily consumption of alcohol $\geq 30 \mathrm{~g}$ per day.

Electrocardiography

Standard 12-lead surface resting ECGs (filter range, 0.5-150 $\mathrm{Hz}, 25 \mathrm{~mm} / \mathrm{s}, 10 \mathrm{~mm} / \mathrm{mV}$ ) were recorded for all the patients. These ECGs were evaluated by cardiologists blinded to the patient data. Heart rate (HR) and QRS duration were noted. Subjects with $f Q R S$ in other leads except inferior leads, QRS duration longer than $120 \mathrm{~ms}$, and incomplete or complete right and left bundle branch block were excluded. fQRS was defined by the presence of various RSR' patterns (QRS duration $<120 \mathrm{~ms}$ ) with or without $Q$ wave, which includes an additional $R$ wave $\left(R^{\prime}\right)$ or notching of the $R$ wave or $S$ wave, or the presence of $>1 R^{\prime}$ fragmentation without typical bundle branch block in 2 contiguous inferior leads (Fig. 1) (5). The standard 12-lead ECG was analyzed without using any magnification.

\section{HIGHLIGHTS}

- Fragmented QRS in lateral leads is shown to be associated with a poor outcome in patients with a known cardiac disease.

- The knowledge about the significance and prevalence of fragmented QRS in inferior leads is scarce.

- The prevalence of fragmented QRS in inferior leads was found to be $21.4 \%$ in this study.

- In this study, we demonstrated the association of fragmented QRS in inferior leads with non-alcoholic fatty liver disease, body mass index, and interventicular septum thickness in young men.

\section{Hepatic ultrasonography}

Hepatic USG was performed by a high-resolution B mode topographic ultrasound system equipped with a convex 3.5 MHz probe (Siemens ACUSON X300 Ultrasound System; Siemens Medical Solutions Inc, Munich, Germany) by a single experienced radiologist blinded to participants' details. Hepatic steatosis was noted. Non-alcoholic fatty liver disease (NAFLD) was diagnosed on the basis of characteristic sonographic features, that is, evidence of diffuse hyperechogenicity of the liver relative to the kidneys, ultrasound beam attenuation, and poor visualization of intrahepatic vessel borders and diaphragm (12).

\section{Transthoracic echocardiography}

Complete 2-dimensional TTE was obtained in all patients. Standard parasternal and apical views were obtained in the left lateral decubitus position using a Vivid 3 Pro ultrasound machine (GE Vingmed Ultrasound, Milwaukee, WI, USA). Images were digitally stored and reviewed by a single cardiologist blinded to the patients' information. The left ventricular ejection fraction (EF), interventricular septum thickness (IVST), and posterior wall thickness (PWT) were measured on $M$-mode traces recorded in the parasternal long axis view. Mitral inflow was assessed from the apical 4-chamber view with pulsed wave Doppler by placing a 1-2 $\mathrm{mm}$ sample volume between the tips of the mitral leaflets during diastole. $\mathrm{E}$ - and $\mathrm{A}$-wave velocities were measured from the mitral inflow profile. Measurements were done according to established standards (13).

\section{Laboratory}

Blood for biochemical analysis was taken after fasting for 12 hours. Routine biochemical investigations and cholesterol parameters of the patients were calculated using the Abbott ARCHITECT c16000 (Abbott Laboratories, Chicago, IL, USA) auto-analyzer. The hematologic tests were performed using the Abbott Cell Dyn Ruby analyzer (Abbott Diagnostics, Chicago, Illinois, USA). Thyroid-stimulating hormone (TSH) test was performed using the chemiluminescent microparticle immunoassay method of the Abbott Architect I 2000 immunology analyzer (Abbott Diagnostics, USA).

\section{Statistical analysis}

Continuous variables were presented as mean \pm standard deviation or median (minimum-maximum), and categorical variables were expressed as number and percentage (\%). The Shapiro-Wilk test was used to identify the normally distributed variables. The continuous variables were compared across the groups using the student's $t$-test or the Mann-Whitney $U$ test. The categorical variables were compared using the chi-square test. To determine the independent predictors of $f Q R S$, multiple logistic regression analysis with enter method was performed. Variables that were found to be statistically significant in the univariate analysis were entered in the regression model. The results of the regression analysis were presented as odds ratio and $95 \%$ confidence interval. All data were analyzed with SPSS version 16.0 for Windows (SPSS Inc., Chicago, IL, USA). The value $\mathrm{p}<0.05$ was considered statistically significant. 


\section{RESULTS}

During the recruitment period, 1,155 consecutive subjects were assessed for enrollment eligibility. We excluded 175 subjects on the basis of predetermined criteria. The eligible 980 participants constituted the final study group. In total, 210 participants with $f Q R S$ in inferior leads were labeled as group 1, whereas the remaining 770 participants without fQRS in inferior leads were labeled as group 2. We found that the prevalence of fQRS in inferior leads was $21.4 \%$ in the whole study population.

Baseline demographic, biochemical, and clinical parameters are shown in Table 1. The mean age, diastolic BP, smoking, glucose, TSH, aspartate aminotransferase, hemoglobin, and sedimentation levels were not different in between the 2 groups. BMI, systolic BP, creatinine, and alanine aminotransferase (ALT) levels were significantly higher in group 1 than in group 2. BMI was $25.9 \pm 2.38 \mathrm{~kg} / \mathrm{m}^{2}$ in group 1 and $24.4 \pm 2.64 \mathrm{~kg} /$ $\mathrm{m}^{2}$ in group $2(\mathrm{p}<0.001)$.

ECG, TTE, and hepatic USG parameters of study groups are shown in Table 2. HR, QRS duration, EF, PWT, E wave, and A wave were not different between the 2 groups. IVST $(12 \pm 0.7$ $\mathrm{mm}$ vs. $11 \pm 0.5 \mathrm{~mm}, \mathrm{p}=0.015$ ) was significantly greater in group 1 than in group 2 .

Of the 980 participants included in the study, 255 (26\%) met the clinical criteria for diagnosis of NAFLD. Group 1 had 98 $(46.7 \%)$ participants with NAFLD. Group 2 had 157 participants with NAFLD (20\%). There were significant differences between the 2 groups in terms of NAFLD percentage $(p<0.001)$. The prevalence of NAFLD in this study was found to be $26 \%$ and is compatible with previous epidemiological studies $(14,15)$.

BMI, IVST, NAFLD, creatinine, ALT, and systolic BP were entered in a model of multiple regression analysis to predict fQRS, a dependent variable. NAFLD was the best independent predictor of fQRS $(\beta=6.115, p=0.001)$. $B M I(\beta=1.448$, $p=0.014)$ and IVST $(\beta=1.058, p=0.029)$ were the other independent predictors of fQRS (Table 3).

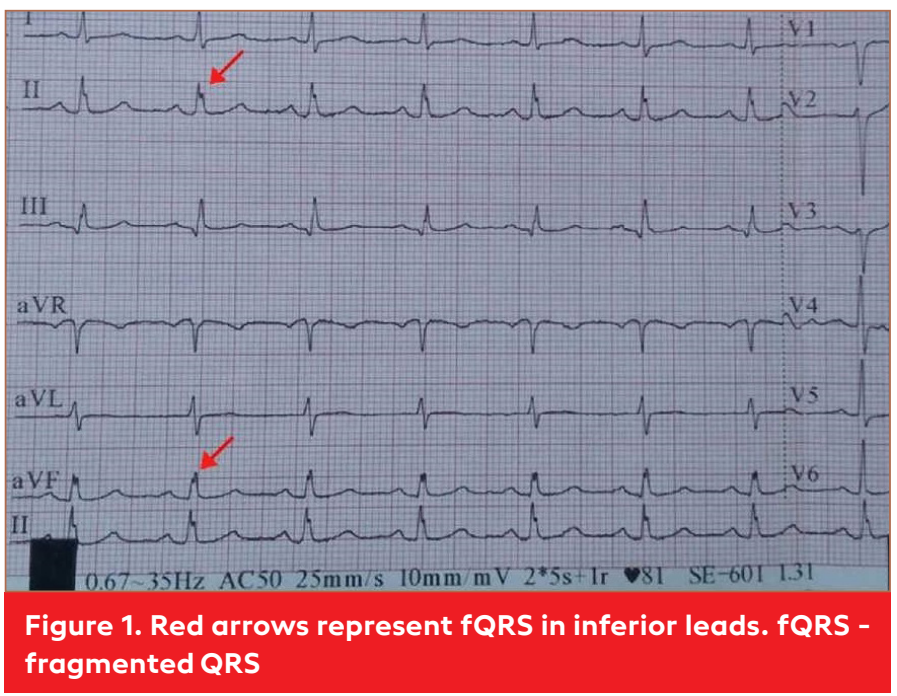

Table 1. Clinical, biochemical, and parameters of study groups

\begin{tabular}{|c|c|c|c|}
\hline & $\begin{array}{l}\text { Group } 1 \\
(n=210)\end{array}$ & $\begin{array}{l}\text { Group } 2 \\
(n=770)\end{array}$ & $P$-value \\
\hline Age, years ${ }^{a}$ & $26.8 \pm 6.3$ & $26.5 \pm 6.2$ & 0.703 \\
\hline $\mathrm{BMI}, \mathrm{kg} / \mathrm{m}^{2 \mathrm{a}}$ & $25.9 \pm 2.38$ & $24.4 \pm 2.64$ & $<0.001$ \\
\hline Systolic BP, mm Hg & $125(20)$ & $120(15)$ & 0.004 \\
\hline Diastolic BP, mm Hg ${ }^{b}$ & $80(10)$ & $75(12)$ & 0.062 \\
\hline Smoking, $\%$ & 39.2 & 40.5 & 0.883 \\
\hline Glucose, mg/dL ${ }^{b}$ & $88(10)$ & $87(10)$ & 0.442 \\
\hline Creatinine, $\mathrm{mg} / \mathrm{dL}^{\mathrm{a}}$ & $0.88 \pm 0.18$ & $0.81 \pm 0.14$ & 0.018 \\
\hline$A L T, U / L^{b}$ & $28(20)$ & $21(12)$ & 0.007 \\
\hline $\mathrm{AST}, \mathrm{U} / \mathrm{L}^{\mathrm{b}}$ & $14(7)$ & $15(7)$ & 0.944 \\
\hline $\mathrm{TSH}, \mathrm{mU} / \mathrm{ml}^{\mathrm{b}}$ & $1.58(1.28)$ & $1.73(1.26)$ & 0.365 \\
\hline Hemoglobin, g/dLa & $13.4 \pm 0.8$ & $13.7 \pm 0.9$ & 0.556 \\
\hline Sedimentation, $\mathrm{m} / \mathrm{hr}^{\mathrm{b}}$ & $3(4)$ & $4(3)$ & 0.074 \\
\hline
\end{tabular}

aean \pm standard deviation.

bMedian (interquartile range).

Group 1: Subjects with fQRS in inferior leads.

Group 2: Men without fQRS in inferior leads.

$P<0.05$ was considered statistically significant.

ALT - alanine aminotransferase; AST - aspartate aminotransferase; BMI - body mass index; BP - blood pressure; TSH - thyroid stimulating hormone.

Table 2. ECG, TTE, and hepatic USG parameters of the study groups

\begin{tabular}{|c|c|c|c|}
\hline & Group 1 & Group 2 & $P$-value \\
\hline $\mathrm{HR}, \mathrm{bpm}^{\mathrm{a}}$ & $80(7)$ & $78(7)$ & 0.618 \\
\hline QRS duration, $\mathrm{ms}^{\mathrm{b}}$ & $92.5 \pm 14.6$ & $90 \pm 15.4$ & 0.842 \\
\hline$E F, \%^{a}$ & $62(5)$ & $63(4)$ & 0.855 \\
\hline IVST, mm & $12 \pm 0.7$ & $11 \pm 0.5$ & 0.015 \\
\hline PWT, mm & $10 \pm 0.5$ & $9.5 \pm 0.5$ & 0.055 \\
\hline E wave, $\mathrm{m} / \mathrm{s}^{\mathrm{b}}$ & $0.8 \pm 0.1$ & $0.75 \pm 0.1$ & 0.376 \\
\hline A wave, $\mathrm{m} / \mathrm{s}^{\mathrm{b}}$ & $0.6 \pm 0.1$ & $0.62 \pm 0.1$ & 0.679 \\
\hline NAFLD, n (\%) & $98(46.7)$ & $157(20)$ & $<0.001$ \\
\hline
\end{tabular}

aedian (interquartile range)

bMean \pm standard deviation.

Group 1: Subjects with fQRS in inferior leads. Group 2: Men without fQRS in inferior leads

$P<0.05$ was considered statistically significant.

ECG - electrocardiography; $E F$ - ejection fraction; $f Q R S$, fragmented QRS; HR - heart rate; IVST - interventricular septum thickness; NAFLD - non-alcoholic fatty liver disease; PWT - posterior wall thickness; TTE

- transthoracic echocardiography; USG - ultrasonography

Table 3. Multiple linear regression model identifying independent factors associated with fQRS

\begin{tabular}{lcc}
\hline Variable & Multiple analysis & \\
\hline IVST & OR $(95 \% \mathrm{CI})$ & $\boldsymbol{P}$-value \\
\hline NAFLD & $1.058(1.006-1.112)$ & 0.029 \\
\hline Creatinine & $6.115(2.582-14.668)$ & 0.001 \\
\hline ALT & $1.034(0.778-1.375)$ & 0.817 \\
\hline BMI & $0.994(0.962-1.026)$ & 0.620 \\
\hline Systolic BP & $1.448(1.115-1.816)$ & 0.014 \\
\hline P 0.05 Was & $1.025(0.965-1.089)$ & 0.416
\end{tabular}

$P<0.05$ was considered statistically significant.

ALT - alanine aminotransferase; BMI - body mass index; BP - blood pressure; $\mathrm{Cl}$ - confidence interval; fQRS - fragmented QRS; IVST interventricular septum thickness, NAFLD - non-alcoholic fatty liver disease; OR - odds ratio. 


\section{DISCUSSION}

In this study, we investigated the prevalence and predictors of $f Q R S$ in inferior leads in young men. First, we found that NAFLD was the best independent predictor of fQRS in inferior leads. Second, BMI and IVST were also independent predictors of $f Q R S$ in inferior leads. Finally, we found that the prevalence of $f Q R S$ in inferior leads in otherwise healthy young men was $21.4 \%$. We believe that these findings could help in diagnosing NAFLD in young men using ECG.

Recent studies have focused on assessing the prognostic significance of $f Q R S$ mainly in patients with CAD, dilated cardiomyopathy, heart failure, hypertension, and cardiac arrhythmias (2-9). However, little is known about the prevalence and significance of fQRS in the general population. The most comprehensive study published to date was conducted by Terho et al. (10) in middle-aged and otherwise healthy population. The prevalence of fQRS was found to be $19.7 \%$ in that study. They concluded that $\mathrm{fQRS}$ is a common finding, especially in the inferior leads without any association with increased risk of mortality in subjects without a known cardiac disease. The findings of this study support those of the aforementioned study in this context. We found the prevalence of fQRS in inferior leads in otherwise healthy young men as $21.4 \%$.

Our study also revealed another important and novel finding. NAFLD was shown to be the best independent predictor of fQRS in inferior leads. Previous studies have shown that NAFLD is associated with different ECG disorders such as atrial fibrillation, conduction defects, and QT prolongation (16). To the best of our knowledge, this was the first time that NAFLD was shown to be associated with $f Q R S$.

NAFLD is the most common chronic liver disease in Western countries and affects approximately $25 \%$ of the adult population. Growing evidence indicates that patients with NAFLD are at substantial risk for the development of hypertension, CHD, cardiomyopathy, and cardiac arrhythmias (17). Since these diseases have been shown to be associated with $F Q R S$ in the literature, it is thought that NAFLD may also be associated with $f Q R S$. Moreover, a recent study showing the relationship between metabolic syndrome and fQRS strengthens the possibility that NAFLD, considered as the hepatic component of the metabolic syndrome, may be associated with fQRS (18). At this point, the novelty of our findings becomes apparent. Our results showed that NAFLD was associated with $f Q R S$ in healthy young men for the first time in literature.

Although early recognition and treatment is crucial, the diagnosis of NAFLD is quite problematic. Value of screening for NAFLD in the community is limited owing to = cost, low predictive value of non-invasive tests, risks of liver biopsy, and lack of effective treatments. Therefore, the National Institute for Health and Care Excellence guideline has recommended screening NAFLD in higher-risk groups with diabetes, metabolic syndrome, and obesity (19). Moreover, measuring hepatic enzymes levels is not very effective in determining NAFLD because the levels can be within normal limits even in advanced disease (20). As a result, most young men with NAFLD will not be diagnosed because they do not fall into the high-risk group if the current guidelines are followed. Therefore, our results are important because they showed that NAFLD was associated with $F Q R S$ in inferior leads in healthy young men. ECG, an easily available medical tool, may aid in the diagnosis of NAFLD in young men earlier in the disease course.

Owing to a combination of shared risk factors, the exact mechanism of increased CVD risk in subjects with NAFLD is difficult to discern (21). Although endothelial dysfunction, oxidative stress, altered lipid metabolism, and insulin resistance all have been extensively studied as an underlying mechanism, recent interest has focused on the other suspects. It has been suggested that myocardial fat accumulation and epicardial adipose tissue (EAT) play an important role in the relationship between NAFLD and CVD $(22,23)$.

EAT, a depot of visceral fat directly overlying the myocardium, is recognized as a metabolically active endocrine organ that secretes adipokines associated with detrimental myocardial and coronary vascular dysfunction $(24,25)$. Although the underlying mechanism is not entirely clear, the association of NAFLD and BMI with $F Q R S$ as shown in our study may be related to EAT. Both NAFLD and high BMI were shown to be related to thicker EAT $(22,23,26)$. Interestingly, a recent magnetic resonance imaging (MRI) study has shown that increased EAT volume index is independently associated with increased myocardial fat accumulation and interstitial myocardial fibrosis (27). Cardiac fibrosis is a well-known underlying mechanism of occurrence of fQRS in various cardiac pathologies. Bekar et al. (28) showed fQRS as an independent predictor of EAT in hypertensive patients in their recent study. Yaman et al. (11) showed that the presence of fQRS was associated with increased EAT in a healthy population. Therefore, we speculate that increased epicardial fat content and its relation to myocardial fibrosis may explain the association of NAFLD and BMI with $\mathrm{FQRS}$ as shown in our study.

Another interesting finding of this study was that IVST was an independent predictor of $\mathrm{fQRS}$ in inferior leads. Accumulating evidence indicates that myocardial structure in left ventricular hypertrophy is affected by myocyte hypertrophy and a progressive accumulation of fibrous tissue within the cardiac interstitium $(29,30)$. The association between myocardial fibrosis and $f Q R S$ was shown in clinical studies $(4,31)$. The results of this study supports previous studies by showing a statistically significant association between $\mathrm{fQRS}$ and IVST.

\section{Study limitations}

Our study had some limitations. First, we enrolled only male subjects because our center serves military personnel. Therefore, it is not possible to draw conclusions for both sexes. Second, we did not measure EAT. We can only speculate about EAT's possible impact on fQRS. To better understand the role of EAT in this process, echocardiography and MRI studies measuring epicardial and myocardial fat content are needed in subjects with NAFLD. Third, we did not include fQRS in the lateral or anterior leads because of the predetermined study protocol. Therefore, it is impossible for us to comment on lateral fQRS, which seems clinically more important. The strength of this study is that a substantial number of otherwise healthy young men can be referred to USG to confirm the diagnosis of NAFLD early in disease course by fQRS in ECG. 


\section{CONCLUSION}

In conclusion, our study showed the association of fQRS in inferior leads with NAFLD, BMI, and IVST in young men. ECG may help in diagnosing NAFLD in otherwise healthy young men.

Conflict of interest: None declared.

Peer-review: Externally peer-reviewed.

Author contributions: Concept - R.S., C.S., Ö.K., G.I.; Design - R.S., C.Ș., Ö.K., G.i.; Supervision - R.S., C.Ș., Ö.K., T.A.; Fundings - None; Materials - R.S., Ö.K., M.O.; Data collection \&/or processing - R.S., C.Ș., Ö.K.; Analysis \&/or interpretation - R.S., C.Ș., T.A.; Literature search - R.S., C.Ș., M.O.; Writing - R.S., C.S. .; Critical review - R.S., C.Ș., G.i.

\section{REFERENCES}

1. Jain $R$, Singh R, Yamini S, Das MK. Fragmented ECG as a risk marker in cardiovascular diseases. Curr Cardiol Rev 2014; 10 : 277-86. [Crossref]

2. Pietrasik G, Zaręba W. QRS fragmentation: diagnostic and prognostic significance. Cardiol J 2012; 19: 114-21. [Crossref]

3. Das MK, Zipes DP. Fragmented QRS: a predictor of mortality and sudden cardiac death. Heart Rhythm 2009; 6 (3 Suppl): S814. [Crossref]

4. Bekar L, Katar M, Yetim M, Çelik O, Kilci H, Önalan O. Fragmented QRS complexes are a marker of myocardial fibrosis in hypertensive heart disease. Turk Kardiyol Dern Ars 2016; 44: 554-60. [Crossref]

5. Das MK, Khan B, Jacob S, Kumar A, Mahenthiran J. Significance of a fragmented $Q R S$ complex versus a $Q$ wave in patients with coronary artery disease. Circulation 2006; 113: 2495-501. [Crossref]

6. Zhang B, Zhen $Y$, Shen D, Zhang G. Significance of fragmented QRS complexes for identifying left ventricular hypertrophy in patients with hypertension. Ann Noninvasive Electrocardiol 2015; 20: 175-80. [Crossref]

7. Sha J, Zhang S, Tang M, Chen K, Zhao X, Wang F. Fragmented QRS is associated with all-cause mortality and ventricular arrhythmias in patient with idiopathic dilated cardiomyopathy. Ann Noninvasive Electrocardiol 2011; 16: 270-5. [Crossref]

8. Pei J, Li N, Gao Y, Wang Z, Li X, Zhang Y, et al. The J wave and fragmented $Q R S$ complexes in inferior leads associated with sudden cardiac death in patients with chronic heart failure. Europace 2012; 14: 1180-7. [Crossref]

9. Oner E, Erturk M, Birant A, Kalkan AK, Uzun F, Avci Y, et al. Fragmented $Q R S$ complexes are associated with left ventricular systolic and diastolic dysfunctions in patients with metabolic syndrome. Cardiol J 2015; 22: 691-8. [Crossref]

10. Terho HK, Tikkanen JT, Junttila JM, Anttonen O, Kenttä TV, Aro $A L$, et al. Prevalence and prognostic significance of fragmented QRS complex in middle-aged subjects with and without clinical or electrocardiographic evidence of cardiac disease. Am J Cardiol 2014; 114: 141-7. [Crossref]

11. Yaman M, Arslan U, Bayramoglu A, Bektas O, Gunaydin ZY, Kaya $A$. The presence of fragmented QRS is associated with increased epicardial adipose tissue and subclinical myocardial dysfunction in healthy individuals. Rev Port Cardiol (Engl Ed) 2018; 37: 469-75. [Article in English, Portuguese] [Crossref]

12. Mehta SR, Thomas EL, Bell JD, Johnston DG, Taylor-Robinson SD. Non-invasive means of measuring hepatic fat content. World J Gastroenterol 2008; 14: 3476-83. [Crossref]
13. Henry WL, DeMaria A, Gramiak R, King DL, Kisslo JA, Popp RL, et al. Report of the American Society of Echocardiography Committee on Nomenclature and Standards in Two-dimensional Echocardiography. Circulation 1980; 62: 212-7. [Crossref]

14. Younossi ZM. Non-alcoholic fatty liver disease - A global public health perspective. J Hepatol 2019; 70: 531-44. [Crossref]

15. Younossi Z, Anstee QM, Marietti M, Hardy T, Henry L, Eslam M, et al. Global burden of NAFLD and NASH: trends, predictions, risk factors and prevention. Nat Rev Gastroenterol Hepatol 2018; 15: 11-20. [Crossref]

16. Ismaiel A, Colosi HA, Rusu F, Dumitrașcu DL. Cardiac Arrhythmias and Electrocardiogram Modifications in Non-Alcoholic Fatty Liver Disease. A Systematic Review. J Gastrointestin Liver Dis 2019; 28: 483-93. [Crossref]

17. Kasper $P$, Martin $A$, Lang $S$, Kütting $F$, Goeser $T$, Demir $M$, et al. NAFLD and cardiovascular diseases: a clinical review. Clin Res Cardiol 2021; 110: 921-37. [Crossref]

18. Bayramoğlu A, Tașolar H, Bektaș O, Yaman M, Kaya Y, Özbilen M, et al. Association between metabolic syndrome and fragmented QRS complexes: Speckle tracking echocardiography study. J Electrocardiol 2017; 50: 889-93. [Crossref]

19. Glen J, Floros L, Day C, Pryke R; Guideline Development Group. Non-alcoholic fatty liver disease (NAFLD): summary of NICE guidance. BMJ 2016; 354: i4428. [Crossref]

20. Rinella ME, Sanyal AJ. Management of NAFLD: a stage-based approach. Nat Rev Gastroenterol Hepatol 2016; 13: 196-205. [Crossref]

21. Stahl EP, Dhindsa DS, Lee SK, Sandesara PB, Chalasani NP, Sperling LS. Nonalcoholic Fatty Liver Disease and the Heart: JACC State-of-the-Art Review. J Am Coll Cardiol 2019; 73: 948-63. [Crossref]

22. lacobellis G, Barbarini G, Letizia C, Barbaro G. Epicardial fat thickness and nonalcoholic fatty liver disease in obese subjects. Obesity (Silver Spring) 2014; 22: 332-6. [Crossref]

23. Petta S, Argano C, Colomba D, Cammà C, Di Marco V, Cabibi D, et al. Epicardial fat, cardiac geometry and cardiac function in patients with non-alcoholic fatty liver disease: association with the severity of liver disease. J Hepatol 2015; 62: 928-33. [Crossref]

24. Sacks HS, Fain JN. Human epicardial adipose tissue: a review. Am Heart J 2007; 153: 907-17. [Crossref]

25. Patel VB, Shah S, Verma S, Oudit GY. Epicardial adipose tissue as a metabolic transducer: role in heart failure and coronary artery disease. Heart Fail Rev 2017; 22: 889-902. [Crossref]

26. Rabkin SW. Epicardial fat: properties, function and relationship to obesity. Obes Rev 2007; 8: 253-61. [Crossref]

27. $\mathrm{Ng} \mathrm{ACT}$, Strudwick M, van der Geest RJ, Ng ACC, Gillinder $L$, Goo SY, et al. Impact of Epicardial Adipose Tissue, Left Ventricular Myocardial Fat Content, and Interstitial Fibrosis on Myocardial Contractile Function. Circ Cardiovasc Imaging 2018; 11: e007372. [Crossref]

28. Bekar L, Kalçık M, Çelik O, Alp Ç, Yetim M, Doğan T, et al. Presence of fragmented QRS is associated with increased epicardial adipose tissue thickness in hypertensive patients. J Clin Ultrasound 2019; 47: 345-50. [Crossref]

29. Díez J, López B, González A, Querejeta R. Clinical aspects of hypertensive myocardial fibrosis. Curr Opin Cardiol 2001; 16: 32835. [Crossref]

30. Weber KT, Brilla CG. Pathological hypertrophy and cardiac interstitium. Fibrosis and renin-angiotensin-aldosterone system. Circulation 1991; 83: 1849-65. [Crossref]

31. Basaran Y, Tigen K, Karaahmet T, Isiklar I, Cevik C, Gurel E, et al. Fragmented QRS complexes are associated with cardiac fibrosis and significant intraventricular systolic dyssynchrony in nonischemic dilated cardiomyopathy patients with a narrow QRS interval. Echocardiography 2011; 28: 62-8. [Crossref] 